\title{
Una propuesta de modelos de datos y protocolos de intercambio de información estandarizados aplicables a sistemas de medida centralizada*
}

\author{
Cristian Fernando Rodríguez ${ }^{* *}$ \\ Bayron Andrés Calvache ${ }^{* * *}$ \\ Eduardo Francisco Caicedo ${ }^{* * * *}$
}

\author{
Recibido: 30/11/2015 • Aceptado: 31/05/2016 \\ DOI: 10.22395/rium.v16n30a8
}

\begin{abstract}
Resumen
En Latinoamérica las estrategias de reducción de pérdidas no técnicas de energía han impulsado la implementación de los sistemas de medición centralizada (SMC). Los SMC, además de incluir las funciones básicas de un sistema AMI, se caracterizan por integrar la medida de múltiples usuarios dentro de una caja diseñada para su instalación en postes, con el fin de restringir el acceso de los usuarios al medidor de energía. En la actualidad la mayoría de los equipos SMC disponibles comercialmente presentan una mezcla de tecnologías, cuyas interfaces y modelos de datos son de carácter propietario, lo que genera un grave problema de interoperabilidad, en parte, originado porque los estándares internacionales están orientados para su aplicación en medidores de energía individuales. Se propone, entonces, en este trabajo un conjunto de estándares al nivel de tecnologías, protocolos de intercambio de información y modelos de datos que pueden ser aplicados en SMC.
\end{abstract}

Palabras clave: infraestructura de medición avanzada, sistema de medición centralizada, AMI, SMC, estándares

* Este artículo hace parte de los resultados obtenidos del proyecto de investigación "Aplicación de un modelo de datos estandarizado para un sistema de medición centralizada de energía eléctrica", financiado por la Universidad del Valle y la empresa Eléctricos del Valle S.A., durante el periodo Agosto 2013 - Diciembre 2015.

** Magister en ingeniería, Universidad del Valle. Investigador, Grupo de Percepción y Sistemas Inteligentes PSI. Escuela de Ingeniería Eléctrica y Electrónica, Facultad de Ingeniería, Universidad del Valle. Correo electrónico: cristian.fernando.rodriguez@correounivalle.edu.co.

*** Magister en ingeniería, Universidad del Valle. Investigador, Grupo de Percepción y Sistemas Inteligentes PSI. Escuela de Ingeniería Eléctrica y Electrónica, Facultad de Ingeniería, Universidad del Valle. Correo electrónico: bayron.calvache@hotmail.com.

***** Doctor en informática industrial, Universidad Politécnica de Madrid. Profesor titular, Escuela de Ingeniería Eléctrica y Electrónica, Facultad de Ingeniería, Universidad del Valle. Director, Grupo de Percepción y Sistemas Inteligentes PSI, Universidad del Valle. Correo electrónico: eduardo.caicedo@correounivalle.edu.co. 


\title{
A proposal for standardized data models and exchange information protocols applicable in centralized metering systems
}

\begin{abstract}
The non-technical energy losses reduction strategies have stimulated the development of centralized metering systems (cms) in Latin America. Furthermore to include ami systems basic functions, the cms embed the measurement of multiple users in a cabinet. To avoid the utility customers getting access to the energy meter, each cabinet is mounted on top of an electrical pole. Currently, the major commercially available cms equipment is setting up with several technologies, which involves owner interfaces and data models. This issue generates an interoperability problem since international standards are based on measurement architectures for the use of individual meters. This paper proposes a set of both information exchange protocols and standardized data models that could be implemented in cms equipments.
\end{abstract}

Key words: advanced metering infrastructure, centralized metering system, ami, cms, standards. 


\section{INTRODUCCIÓN}

En Colombia y países en desarrollo, los sistemas de medición centralizada (SMC) se definen como un esquema de medición de energía eléctrica que, además de incluir las funciones básicas de un sistema AMI, se caracteriza por integrar y centralizar la medida de múltiples usuarios dentro de un único gabinete o caja diseñada para su instalación en postes. Esto, con el objetivo de reducir las pérdidas no técnicas de energía eléctrica, ocasionadas por conexiones ilegales a la red eléctrica, alteración de medidores y errores administrativos de las empresas de servicios públicos, a través de la proporción de un mejor control y menor riesgo al momento de realizar los procesos de corte, reconexión y facturación de energía, así como restringir el acceso por parte de los usuarios a las acometidas del medidor y al medidor mismo [1].

Dentro de un SMC los modelos de datos definen la forma en que se estructura la información, sin que esta dependa de su medio de transporte o protocolo de comunicación. En la actualidad existen especificaciones internacionales orientadas a estandarizar dichos modelos incluyendo la capa de aplicación utilizada para transportarlos. Entre estas se destacan el ANSI C12.19 y ANSI C12.22 [2], del Instituto Nacional Estadounidense de Estándares (ANSI), así como la suite DLMS/COSEM [3], elaborada por la Comisión Electrotécnica Internacional (IEC). A pesar de ello, la mayoría de los equipos SMC disponibles comercialmente presentan una mezcla de tecnologías, cuyas interfaces y modelos de datos son de carácter propietario, lo que agrava el problema de interoperabilidad, de los sistemas AMI del país, en parte, porque los estándares internacionales están orientados para su aplicación en medidores de energía individuales [4-5].

A partir de lo planteado, en el presente artículo se propone una serie de estándares abiertos de modelos de datos y protocolos de intercambio de información para su posible aplicación sobre SMC. Para esto, en la sección 1, se introduce la arquitectura del SMC, haciendo énfasis en los elementos e interfaces de comunicación que la conforman, y se realiza una revisión de las tecnologías y estándares internacionales más usados en la práctica. En la sección 2 se clasifican y proponen los que pueden utilizarse sobre los $\mathrm{SMC}$. En la sección 3 se muestra una discusión del trabajo y, por último, en la sección 4 , se exponen las conclusiones finales.

\section{MATERIALES Y MÉTODOS}

\subsection{Arquitectura SMC}

En la figura 1 se muestra la arquitectura general de un SMC, que típicamente se compone de 4 elementos: 


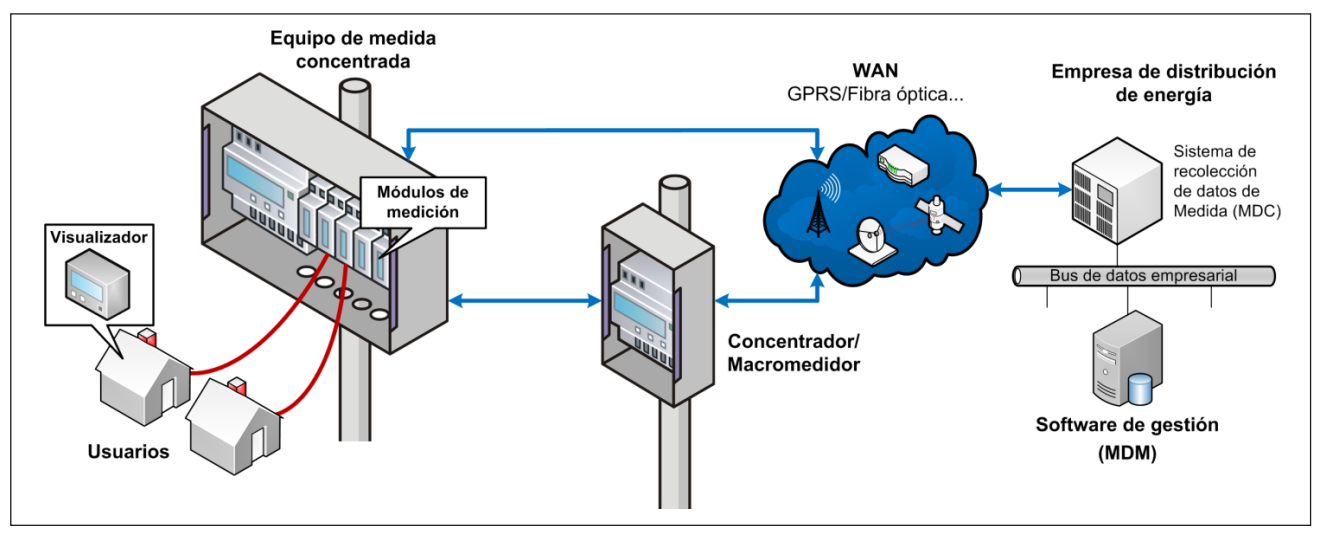

Figura 1. Arquitectura del SMC.

Fuente: elaboración propia

- Equipo medida concentrada.

- Concentrador/macromedidor.

- Visualizador.

- Software de gestión.

\subsubsection{Componentes}

\section{Equipo de medida concentrada (EMC)}

Los equipos de medición concentrada (EMC) son dispositivos de medición inteligente que integran la medida de múltiples usuarios. Generalmente están conformados por una unidad de procesamiento central (colector de datos), módulos de comunicación, módulos de medición de energía eléctrica (unidades de medida) y dispositivos de corte y reconexión. Pueden ser accedidos desde el centro de gestión a través del concentrador/ macromedidor o directamente mediante tecnologías WAN como GSM/UMTS, Fibra Óptica, Ethernet, enlaces microonda, etc.

\section{Concentrador/macromedidor}

El concentrador es un elemento a través del cual se gestiona la información del equipo de medida concentrada, y sirviendo, además, de interfaz con el centro de gestión. De forma opcional puede ejercer funciones de macromedidor que permiten obtener diferentes variables (como energía activa, reactiva y potencia suministrada por fase) de los transformadores que conforman las redes de distribución, con el fin de calcular balances de consumos para la detección de zonas con posible fraude. 


\section{Visualizador}

El visualizador es un dispositivo que permite desplegar en una pantalla la información de los registros del medidor (energía, alarmas, eventos, mensajes, códigos de error, etc.), para el usuario del servicio en el interior de su predio. Normalmente recibe la información desde el equipo de medida concentrada utilizando un protocolo y un medio de comunicación definido (par trenzado, inalámbrico o PLC); sin embargo, es factible también el uso de medios como Internet, telefonía, televisión IP o red celular para transmitir la información directamente desde el centro de gestión, eliminando la necesidad de este dispositivo.

\section{Software de gestión}

El software de gestión es una aplicación instalada en el centro de gestión para la administración, registro, visualización y análisis de consumos, eventos y alarmas de todos los elementos que componen el SMC. Se apoya del sistema de recolección de datos de medida (MDC) para obtener la información de los EMC y los concentradores de forma periódica o por demanda.

\subsubsection{Interfaces}

En la figura 2 se muestran las interfaces comunes presentes en la arquitectura SMC. En total se identifican 5 tipos los cuales se describen a continuación:

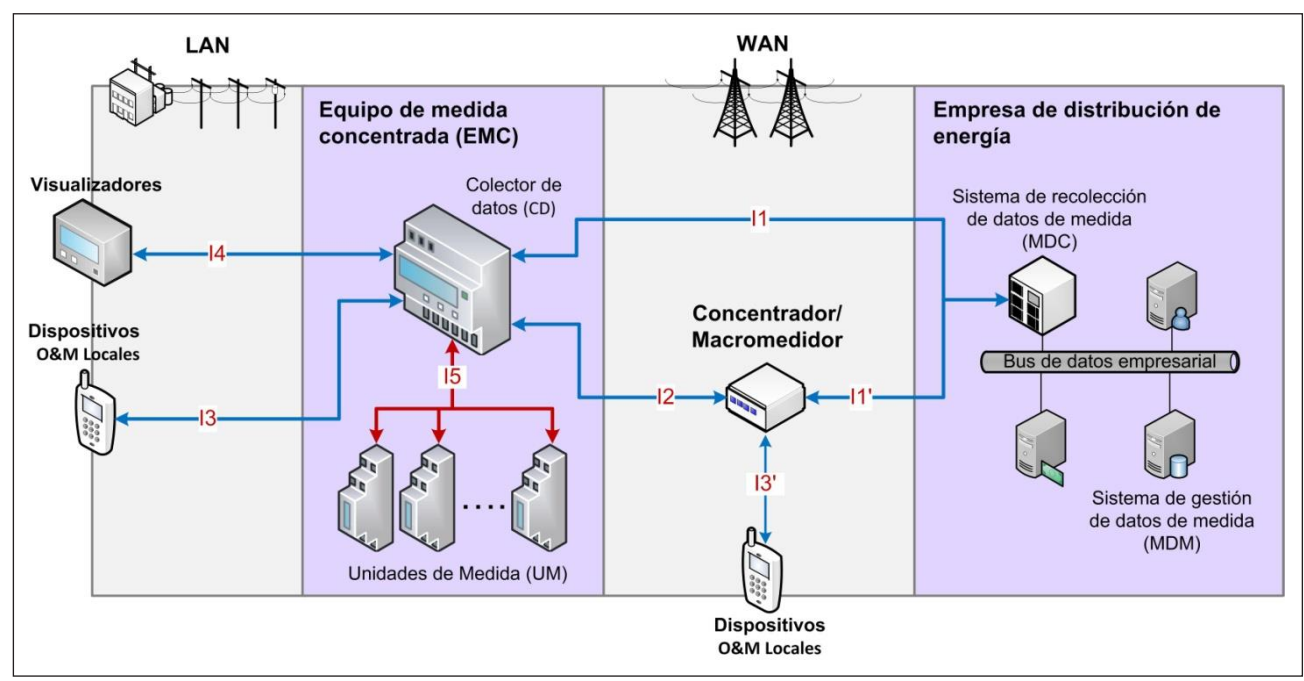

Figura 2. Interfaces del SMC.

Fuente: elaboración propia 
- Interfaz I1: comunica el EMC y el concentrador con el centro de gestión.

- Interfaz I2: comunica el EMC con el concentrador.

- Interfaz I3: comunica el EMC y el concentrador con los dispositivos de operación y mantenimiento locales (Handhelds, celulares o PCs), para las tareas de configuración y mantenimiento cuando no puedan realizarse de forma remota.

- Interfaz I4: comunica el EMC con los dispositivos visualizadores.

- Interfaz I5: comunica, en el interior del EMC, el colector de datos (CD) con las unidades de medida (UM).

\subsection{Estándares internacionales}

Para entrar en contexto con la estandarización al nivel internacional, a continuación se realiza una revisión bibliográfica de las tecnologías y especificaciones con mayor presencia en la práctica.

\subsubsection{ANSI C12.18}

El ANSI C12.18 [6] es un estándar que define las capas física, enlace de datos y aplicación para el intercambio de información con medidores de energía mediante puerto óptico. Provee varios servicios que permiten el transporte total o parcial de las tablas de información, definidas en el estándar ANSI C12.19, soportado sobre un esquema de permisos de acceso. En la actualidad ANSI C12.18 se encuentra ampliamente difundido en los medidores de energía eléctrica, con aplicación exclusiva sobre puertos locales.

\subsubsection{ANSI C12.22}

ANSI C12.22 [2] es un estándar abierto que describe un protocolo para el transporte de tablas de datos ANSI C12.19 a través de redes de comunicaciones y puertos ópticos locales. Soporta protocolos TCP-UPD/IP sobre Ethernet y PPP (Point-to-Point Protocol), además de redes celulares sobre GSM/UMTS. En términos de seguridad maneja un esquema de permisos de acceso con soporte opcional de servicios de encriptación.

\subsubsection{ANSI C12.19}

ANSI C12.19 [5] es un estándar modelo de datos compuesto por un conjunto de tablas, uniformes, estructuradas y adaptativas, agrupadas en secciones llamadas décadas. Cada década asocia características y funciones similares, como tiempo de uso, perfiles de carga e identificadores, entre otros. La información de las tablas es transmitida total o parcialmente, hacia o desde un dispositivo final (medidor energía), utilizando 
servicios de escritura o lectura proporcionados por los protocolos de intercambio de información que soporten el presente modelo de datos.

\subsubsection{BACnet}

BACnet [7] es un protocolo de comunicaciones para la implementación de redes de control y automatización. Es un estándar ISO para su aplicación en HVAC (heating, ventilating and air conditioning), iluminación, seguridad, detección de incendios e interfaces de compañías de servicios públicos. Soporta un gran número de tecnologías y emplea un modelo de datos orientado a objetos que proveen información para el control de carga y facturación de energía [8].

\subsubsection{IEC 61334-5-1 S-FSK PLC}

El IEC 61334-5-1 S-FSK [9] es parte de la suite de estándares IEC 61334, desarrollada por IEC TC 57 WG09 a finales de los 90. Es una tecnología PLC de banda angosta, dentro del espectro A CENELEC, que utiliza modulación de propagación por desplazamiento de frecuencia (S-FSK) para proporcionar una comunicación robusta, con una alta relación señal a ruido, a costa de una baja velocidad de transferencia. Puede integrarse con cualquier capa de aplicación, incluyendo la definida por DLMS/COSEM.

\subsubsection{IEC 61850}

IEC 61850 [10] es una suite de estándares que describen modelos y funciones, de subestaciones y centros de control, para tareas relacionadas con recursos de energía distribuidos (DER), gestión de la demanda, distribución automatizada y servicios de medida. Su modelo de datos organiza la información en una jerarquía de objetos, compuestos por tipos de datos estandarizados, atributos comunes, clases de datos comunes, objetos de datos, nodos lógicos (LN) y dispositivos lógicos (LD).

\subsubsection{IEC 62056-21 "FLAG"}

El IEC 62056-21 [11], también conocido como "protocolo Flag", es un estándar para la lectura y programación de medidores de energía eléctrica mediante acceso local o remoto. Principalmente opera sobre puerto óptico, corriente de lazo, interfaces eléctricas V.24/V.28 y módems PSTN y GSM/UMTS. En los modos A, B, C y D, la capa de aplicación del IEC 62056-21 organiza la información en forma de direcciones de memoria definidas por el fabricante del dispositivo. El modo E por su lado solo provee un medio para acceder a los atributos, métodos y objetos de la suite DLMS/COSEM mediante el perfil HDLC. 


\subsubsection{IEC 62056-3-1 Euridis}

El IEC 62056-3-1 [12] es una solución sencilla, confiable y de bajo costo para la lectura local y remota de medidores. Consiste en un bus de cable par trenzado, donde se conectan hasta 100 medidores, y un acoplador magnético para el intercambio de información utilizando una Handheld. Aunque Euridis permite soportar funciones AMR local y AMR remota, solo especifica las capas bajas del modelo OSI sin indicar un modelo de datos. No obstante, DLMS/COSEM lo ha incluido como uno de sus perfiles de comunicación para permitir la operación de sus servicios y objetos.

\subsubsection{DLMS/COSEM: IEC 62056}

DLMS/COSEM [13] es una suite de estándares internacionales abiertos para la comunicación con medidores de cualquier tipo de energía o dispositivos inteligentes. Desarrollado al final de los 90, tiene como objetivo proporcionar un medio para el intercambio de datos estandarizados e interoperables sobre un amplio rango de perfiles de comunicación. Para cumplir estos objetivos, DLMS/COSEM divide el problema en 3 pasos: modelado de la información del medidor, servicios para acceder el modelo de datos y transporte de las unidades de datos del protocolo de aplicación (APDU) a través de canales de comunicación. Su modelo de datos está basado en una biblioteca de clases de interfaz COSEM junto a un sistema de identificación de objetos OBIS.

\subsubsection{M-BUS: EN 13757}

M-Bus (Meter-Bus) [14] es una suite de estándares europeos de bajo costo para la lectura local o remota de medidores de energía, gas o agua. Basado en una arquitectura maestro/esclavo, define un bus de cable par trenzado con señalización de banda base (sin portadora), una interfaz inalámbrica en las bandas 169,433 y $868 \mathrm{MHz}$ y un bus alternativo para sistemas pequeños de hasta 5 metros. Con relación a su aplicación, M-Bus es un protocolo orientado al AMR local y AMR remoto, con soporte de la suite DLMS/COSEM.

\subsubsection{MODBUS}

MODBUS [15] es un estándar que define un protocolo de mensajes de capa de aplicación, para proveer comunicación cliente/servidor entre dispositivos conectados sobre enlaces seriales asíncronos RS232 y RS485 o redes TCP/IP sobre Ethernet. De forma similar al IEC 62056-21, su capa de aplicación está definida por una serie de direcciones de memoria o registros, cuya organización es especificada y mantenida por el fabricante del dispositivo. 


\subsubsection{Smart message language (SML)}

SML [16] es un protocolo de comunicación desarrollado y aplicado por una serie de empresas públicas europeas (RWE, EON, EnBW, Vattenfall). Define una estructura simple, basada en archivos, para su implementación en sistemas embebidos de bajo consumo, que facilita el intercambio de datos sobre enlaces de gran distancia. SML especifica también un modelo de datos y una capa de aplicación, la cual es independiente del medio de comunicación, así como un protocolo de transporte que puede aplicarse de forma opcional.

\subsubsection{Meters and more}

Meters and more [17] es una especificación abierta que define una arquitectura basada en medidores inteligentes, concentradores y una estación central. Emplea una solución PLC basada en una versión mejorada de LONTalk o su propio protocolo SMITP BPSK de banda angosta. Al nivel de capa de aplicación, implementa funciones de autodetección de medidores, auto-configuración de la red, encriptación, autenticación y protección contra ataques playback. Aunque define su propio modelo de datos basado en tablas de atributos homogéneos, DLMS/COSEM incluye esta tecnología dentro de sus perfiles de comunicación.

\subsubsection{Prime}

PRIME [18] es un estándar PLC tipo OFDM que proporciona una solución pública, abierta y libre de patentes para sistemas de transmisión de datos sobre la banda $\mathrm{A}$ CENELEC [4]. Provee modos de transmisión robusta, velocidades de transmisión de hasta 130kbps y compatibilidad con aplicaciones IPv6. La suite DLMS/COSEM incluye PRIME como parte de sus perfiles de comunicación, permitiendo soporte completo de sus servicios y objetos.

\subsubsection{ZIGBEE SmartEnergyProfile}

ZIGBEE es una tecnología inalámbrica de baja potencia diseñada para la monitorización y control de dispositivos en redes de área personal (WPAN) o redes área doméstica (HAN). Trabaja sobre el estándar IEEE 802.15.4, en las bandas ISM (Industrial, Scientific and Medical) 2.4GHz o 915/868 MHz, con soporte de topología tipo Mesh para extender el rango de los dispositivos. SmartEnergyProfile [19] es un perfil de capa de aplicación que define descripciones de dispositivo para lectura de medidores, respuesta de demanda, medidores prepago y gestión de carga con el fin de fomentar un entorno de interoperabilidad. 


\subsubsection{LOWPAN}

6LoWPAN [20-22] es un protocolo para el transporte de información sobre redes Mesh inalámbricas IEEE 802.15.4, desarrollado para conectar a Internet dispositivos embebidos a través de la simplificación de la funcionalidad IPv6. De forma similar a las aplicaciones de redes LAN actuales, 6LoWPAN utiliza una interfaz basada en sockets donde cada aplicación puede asociarse con un protocolo TCP o UDP.

\subsubsection{DL/T645}

El DL/T645 [23] es un protocolo de origen chino que define la comunicación entre medidores de energía y equipos terminales de datos (Handhelds o Displays). Maneja una arquitectura maestro-esclavo en modo punto a punto y punto-multipunto, y establece las capas física, enlace de datos y aplicación del modelo de referencia OSI. Puede utilizarse también con módulos PLC tipo BFSK para soportar aplicaciones de medición prepago.

\subsubsection{G3-PLC}

G3-PLC [24] es un estándar, desarrollado por Sagem, ERDF y Maxim, que emplea multiplexación OFDM para proveer una alta resistencia ante interferencias y atenuaciones. Puede establecer enlaces de confiables de hasta 9 kilómetros, al tiempo que cruza transformadores de media tensión. Al igual que PRIME, la suite DLMS/COSEM ha incluido esta tecnología como parte de sus perfiles de comunicación, permitiendo soporte completo de sus servicios y objetos.

\subsubsection{ITU-T G.hn Broadband}

G.hn [25] es un estándar desarrollado por la International Telecommunications Union (ITU), que permite establecer redes residenciales sobre cualquier tipo de medio cableado, sea línea de potencia, par trenzado telefónico o cable coaxial, con velocidades de hasta $1 \mathrm{Gbit} / \mathrm{s}$. Especifica una capa física única basada en multiplexación FFT OFDM e incluye la capacidad de atenuar frecuencias específicas para evitar interferencias con bandas de radio aficionadas, así como otros servicios de radio licenciados.

\section{RESULTADOS Y PROPUESTA DE ESTÁNDARES Y TECNOLOGÍAS DE COMUNICACIÓN PARA SU USO EN SMC}

Dado el gran número de estándares observados en la sección 1.2, es necesario reducir el número de opciones disponibles. Para esto se plantea a continuación un proceso de clasificación, selección y propuesta de las especificaciones antes mencionadas. 
Antes de continuar, se debe tener presente que para facilitar la interoperabilidad, los SMC requieren estándares y tecnologías cuya aplicación esté orientada a los sistemas AMI. Por lo tanto, especificaciones como BACnet e IEC61850, al estar exclusivamente relacionados con la Automatización del Hogar y las subestaciones, se consideran no apropiadas para SMC, ya que el uso de estos estándares, para fines distintos a los que fueron diseñados, genera complejas adaptaciones que reducen la interoperabilidad y vuelven el sistema de carácter propietario.

\subsection{Clasificación por interfaces}

La clasificación por interfaces tiene como objetivo identificar los estándares que pueden ser aplicados en los diferentes enlaces de comunicación de los SMC. Para esto, primero se definen, de acuerdo con [26], los rangos de cobertura que requieren todas las interfaces, para luego asociarlos con las especificaciones antes revisadas. El resultado se observa en la figura 3, donde a la clasificación se le han adicionado varias tecnologías fundamentales, como WIFI, Bluetooth, fibra óptica y Ethernet, para complementar a las ya vistas en la sección 1.2.

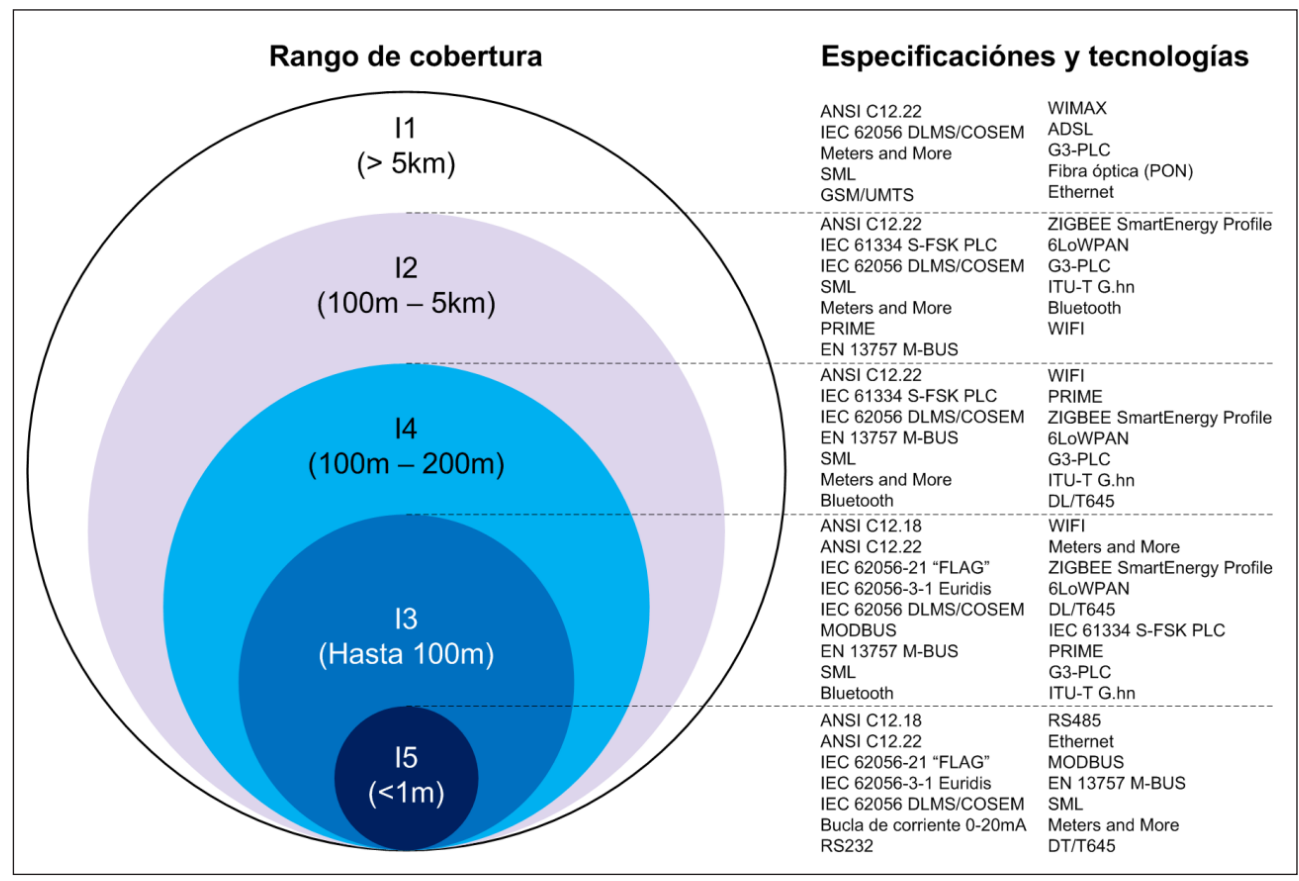

Figura 3. Clasificación de estándares con base a la interfaz. Fuente: elaboración propia 


\subsection{Selección de estándares}

Para lograr reducir las pérdidas no técnicas de energía eléctrica, los SMC deben cumplir una serie de requerimientos especiales de comunicación. Dichos requerimientos se definen en la tabla 1. Como se observa, en todos los casos se le da importancia a garantizar un intercambio de datos robusto, seguro y soportado bajo un modelo de datos.

Tabla 1. Requerimientos de las tecnologías y protocolos para SMC

\begin{tabular}{|c|c|c|}
\hline & Requerimiento & Justificación \\
\hline \multirow{3}{*}{ Todos } & $\begin{array}{l}\text { Robusto intercambio } \\
\text { de datos }\end{array}$ & $\begin{array}{l}\text { Los SMC se instalan y operan a la intemperie. Por lo tanto, están } \\
\text { expuestos a altas temperaturas, ambientes corrosivos y todo tipo de } \\
\text { interferencias que afectan las comunicaciones. }\end{array}$ \\
\hline & $\begin{array}{l}\text { Soporte de seguridad y } \\
\text { encriptación }\end{array}$ & $\begin{array}{l}\text { La información del SMC siempre se debe proteger. Por lo tanto, todas } \\
\text { las comunicaciones deben ser autenticadas y encriptadas para evitar } \\
\text { cualquier intervención o espionaje de las interfaces. }\end{array}$ \\
\hline & $\begin{array}{l}\text { Soporte de modelo de } \\
\text { datos maduro y mante- } \\
\text { nido por un organismo } \\
\text { internacional }\end{array}$ & $\begin{array}{l}\text { El modelo de datos en los SMC permite que equipos de diferentes } \\
\text { fabricantes puedan comunicarse entre sí, presentando la información } \\
\text { de forma coherente y estandarizada. En I5 no es relevante su soporte } \\
\text { debido a que la interfaz está integrada dentro del EMC. }\end{array}$ \\
\hline \multirow{3}{*}{ I5 } & $\begin{array}{l}\text { Velocidad de transmi- } \\
\text { sión baja }\end{array}$ & $\begin{array}{l}\text { El volumen de información, que las UM intercambian con el CD, } \\
\text { generalmente es pequeño (150 Bytes promedio por lectura). No se } \\
\text { requiere un canal con una gran velocidad de datos. }\end{array}$ \\
\hline & $\begin{array}{l}\text { Tecnologías de comu- } \\
\text { nicación basadas en } \\
\text { un bus de datos serial } \\
\text { sobre par trenzado. }\end{array}$ & $\begin{array}{l}\text { Se garantiza un enlace dedicado y aislado del exterior. Consumen } \\
\text { menos energía, son menos susceptibles a interferencias por ruidos, } \\
\text { son económicos de implementar y su hardware es sencillo. }\end{array}$ \\
\hline & $\begin{array}{l}\text { Soporte de más de } 12 \\
\text { nodos }\end{array}$ & Un CD se comunica típicamente con 12 o más UM. \\
\hline \multirow{3}{*}{ I4 } & $\begin{array}{l}\text { Velocidad de transmi- } \\
\text { sión baja }\end{array}$ & $\begin{array}{l}\text { El volumen de información que el EMC intercambia con el visuali- } \\
\text { zador generalmente es pequeño ( } 150 \text { Bytes promedio por lectura). No } \\
\text { se requiere un canal con una gran velocidad de datos. }\end{array}$ \\
\hline & $\begin{array}{l}\text { Tecnologías de comu- } \\
\text { nicación basadas en RF } \\
\text { o PLC }\end{array}$ & $\begin{array}{l}\text { Las tecnologías RF y PLC requieren un mantenimiento menor que } \\
\text { el par trenzado. Además, se protege al usuario y al EMC de algún } \\
\text { corto circuito que se pueda generar en el enlace. }\end{array}$ \\
\hline & $\begin{array}{l}\text { Soporte de más de } 12 \\
\text { nodos }\end{array}$ & $\begin{array}{l}\text { Un EMC se comunica típicamente con } 12 \text { o más visualizadores, cada } \\
\text { uno asociado a una UM. }\end{array}$ \\
\hline
\end{tabular}


Una propuesta de modelos de datos y protocolos de intercambio de información estandarizados aplicables a ... 161

\begin{tabular}{|c|c|c|}
\hline & Requerimiento & Justificación \\
\hline $\mathrm{I} 3$ & $\begin{array}{l}\text { Velocidad de transmi- } \\
\text { sión media }\end{array}$ & $\begin{array}{l}\text { Los dispositivos de O\&M pueden llegar a manejar un volumen de } \\
\text { datos importante al realizar las operaciones de configuración y des- } \\
\text { carga de información del EMC o concentrador. }\end{array}$ \\
\hline \multirow[t]{2}{*}{ I3 } & $\begin{array}{l}\text { Tecnologías de comu- } \\
\text { nicación basadas en RF }\end{array}$ & $\begin{array}{l}\text { Para zonas de difícil gestión, se garantiza facilidad de acceso, hasta } \\
100 \mathrm{~m} \text {, a los EMC y concentradores. Por ser un enlace punto a punto, } \\
\text { son más económicos de implementar frente al PLC. Además, se aísla } \\
\text { la interfaz para evitar que algún corto circuito se propague por el par } \\
\text { trenzado y genere graves daños al operador y los equipos. }\end{array}$ \\
\hline & Soporte de 1 nodo & $\begin{array}{l}\text { La comunicación punto a punto no requiere el soporte de múltiples } \\
\text { nodos. }\end{array}$ \\
\hline \multirow{3}{*}{$\mathrm{I} 2$} & $\begin{array}{l}\text { Velocidad de transmi- } \\
\text { sión alta. }\end{array}$ & $\begin{array}{l}\text { El volumen de información que maneja el concentrador se incrementa } \\
\text { con cada EMC que tenga asociado y con los periodos de recolección } \\
\text { de datos [27]. }\end{array}$ \\
\hline & $\begin{array}{l}\text { Tecnologías de comu- } \\
\text { nicación basadas en RF } \\
\text { o PLC }\end{array}$ & $\begin{array}{l}\text { Son más económicos de implementar, frente al par trenzado, por los } \\
\text { amplios rangos de cobertura manejados. }\end{array}$ \\
\hline & $\begin{array}{l}\text { Soporte de más de } 100 \\
\text { nodos }\end{array}$ & $\begin{array}{l}\text { Un concentrador, dependiendo de la arquitectura, puede asociar hasta } \\
100 \text { puntos de medida. }\end{array}$ \\
\hline \multirow[b]{2}{*}{ I1 } & $\begin{array}{l}\text { Alta velocidad de } \\
\text { transmisión }\end{array}$ & $\begin{array}{l}\text { El volumen de información es alto, debido a que se transportan los } \\
\text { datos de todos los EMC y los concentradores. }\end{array}$ \\
\hline & $\begin{array}{l}\text { Tecnologías de comu- } \\
\text { nicación asociadas a } \\
\text { WAN }\end{array}$ & $\begin{array}{l}\text { Cualquier tecnología que maneje una cobertura de más de } 5 \mathrm{~km} \text {, con } \\
\text { una alta velocidad de transmisión, se considera adecuada. }\end{array}$ \\
\hline
\end{tabular}

Fuente: elaboración propia

En resumen, el volumen de datos transportado en la arquitectura siempre crece a medida que la información viaja hacia el centro de gestión, hecho que exige el uso de canales con una velocidad de transmisión alta comparada con la que necesitan las UM con el CD. Por su parte, el número de nodos requeridos por las interfaces cambia según la cantidad de dispositivos que intervengan en la comunicación. Finalmente, las tecnologías sugeridas en cada caso responden a la relación costo-beneficio que se puede obtener de su implementación.

Aplicando los requerimientos de la tabla 1 sobre los estándares de la figura 3, se obtiene la figura 4. Se concluye que no existe una única tecnología que cobije todas las necesidades de comunicación, por lo que su selección dependerá de las características del entorno donde se instalen los SMC y la inclinación de la empresa de servicios públicos en el uso de algunas en especial. 


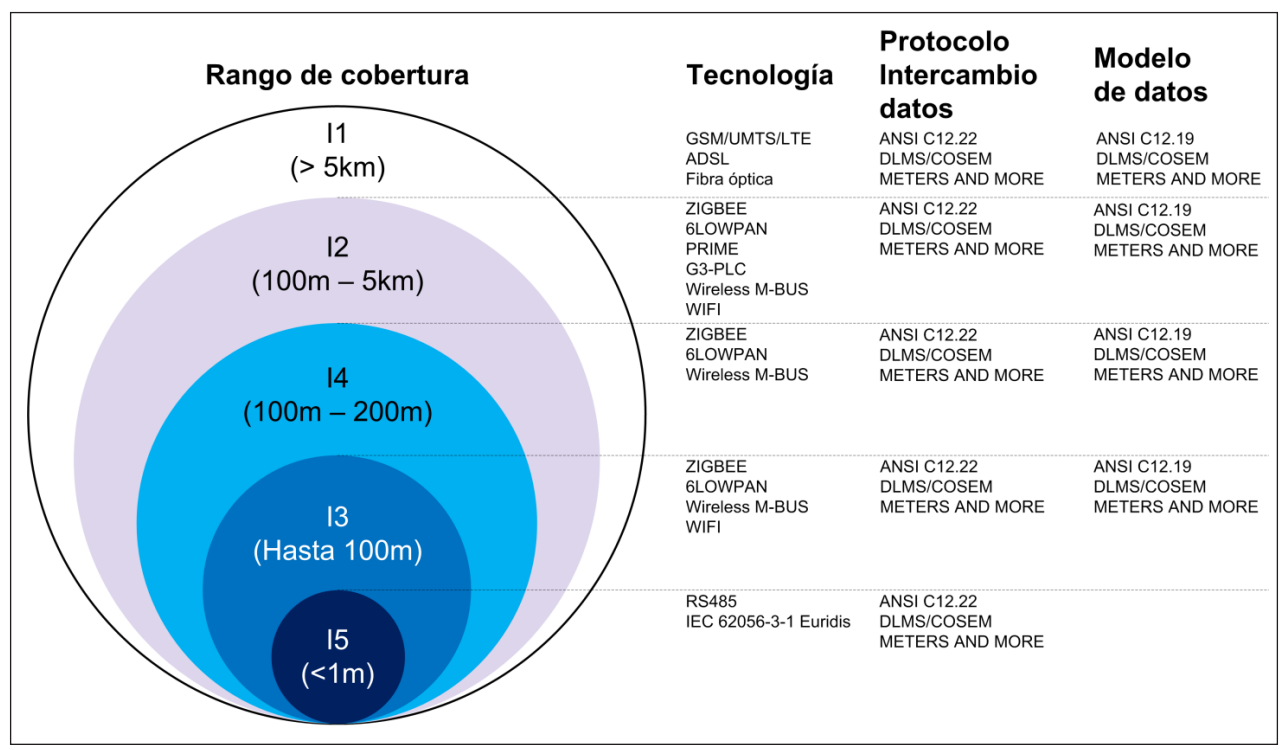

Figura 4. Selección de tecnologías y estándares.

Fuente: elaboración propia

Al nivel de protocolos de intercambio de información, se presentan, como opciones ANSI C12.22, DLMS/COSEM y Meters and more. Dichos estándares son los únicos en la lista que cumplen los requisitos de seguridad, y manejan, cada uno, un modelo de datos estandarizado y maduro.

En modelo de datos se encontraron como aptos ANSI C12.19, DLMS/COSEM y Meters and More. Estos modelos se encuentran soportados por organismos internacionales y tienen la capacidad de ser transportados sobre varios medios de comunicación. Sin embargo, para ser coherentes con el planteamiento del artículo, se debe elegir uno de los tres, siguiendo los requerimientos establecidos en la tabla 2.

Tabla 2. Requerimientos de los modelos de datos para SMC

\begin{tabular}{|l|l|}
\hline \multicolumn{1}{|c|}{ Requerimiento } & \multicolumn{1}{c|}{ Justificación } \\
\hline $\begin{array}{l}\text { Soporte de otros servicios } \\
\text { como agua, gas o calor }\end{array}$ & $\begin{array}{l}\text { Para que el modelo de datos se mantenga a futuro, debe ser abierto a } \\
\text { otros tipos de servicios, diferentes a energía. }\end{array}$ \\
\hline $\begin{array}{l}\text { Descarga del modelo de } \\
\text { datos }\end{array}$ & $\begin{array}{l}\text { Los equipos, cuyo modelo de datos sea desconocido, deben ofrecer la } \\
\text { opción de descargar dicho modelo. Esto permite conocer la estructura } \\
\text { lógica interna de un equipo, aunque no se disponga de su manual o su } \\
\text { software. }\end{array}$ \\
\hline
\end{tabular}




\begin{tabular}{|l|l|}
\hline $\begin{array}{l}\text { Soporte de la actualización } \\
\text { de Firmware }\end{array}$ & $\begin{array}{l}\text { La actualización del Firmware es importante para la mejora funcional } \\
\text { de los equipos que conforman los SMC. }\end{array}$ \\
\hline $\begin{array}{l}\text { Permite extender sus tablas/ } \\
\text { objetos/estructuras }\end{array}$ & $\begin{array}{l}\text { La extensión del modelo de datos permite enriquecer la estructura lógica } \\
\text { de un equipo mediante la adición de tablas u objetos que el fabricante } \\
\text { considere necesario. }\end{array}$ \\
\hline
\end{tabular}

Fuente: elaboración propia

Aplicado los requerimientos de la tabla 2 sobre los modelos de datos seleccionados, se obtiene la tabla 3. De ella se observa que el estándar que cuenta con el mayor grado de conformidad es DLMS/COSEM.

Tabla 3. Conformidad de los modelos de datos con los requerimientos planteados

\begin{tabular}{|l|c|c|c|}
\hline \multicolumn{1}{|c|}{ Tipo modelo. } & ANSI C12.19 & DLMS/COSEM & Meters and More \\
\hline $\begin{array}{c}\text { Tablas agrupadas } \\
\text { en parámetros } \\
\text { homogéneos. }\end{array}$ & $\begin{array}{c}\text { Objetos instanciados } \\
\text { partir de clases. }\end{array}$ & $\begin{array}{c}\text { Tablas agrupadas } \\
\text { en parámetros } \\
\text { homogéneos. }\end{array}$ \\
\hline $\begin{array}{l}\text { Permite extender el } \\
\text { modelo. }\end{array}$ & Sí. & Sí. & Sí. \\
\hline $\begin{array}{l}\text { Soporta medición } \\
\text { multiservicios. }\end{array}$ & Sí. & Sí. & No. \\
\hline $\begin{array}{l}\text { Soporta descarga del } \\
\text { modelo de datos }\end{array}$ & Sí. & Sí. & Sí. \\
\hline $\begin{array}{l}\text { Soporta actualización } \\
\text { de Firmware }\end{array}$ & No especificado & Sí. & \\
\hline
\end{tabular}

Fuente: elaboración propia

\subsection{Propuesta de estándares}

La selección final de estándares se ilustra en la figura 5. De este resultado es importante destacar que la mayoría de tecnologías, como PRIME, G3-PLC, ZIGBEE, M-BUS, Euridis y WIFI, están incluidas dentro de los perfiles de comunicaciones de DLMS/ COSEM y que este estándar ha sido recomendado por el ICONTEC, a través de la Norma Técnica Colombiana NTC-6079, para su uso en los sistemas AMI del país.

\section{DISCUSIÓN}

Gran parte de los estándares internacionales han sido planteados para la medida individual de energía eléctrica, y no para los SMC. Esto se debe a que los países donde rigen estas especificaciones generalmente son desarrollados y el fenómeno de pérdidas 


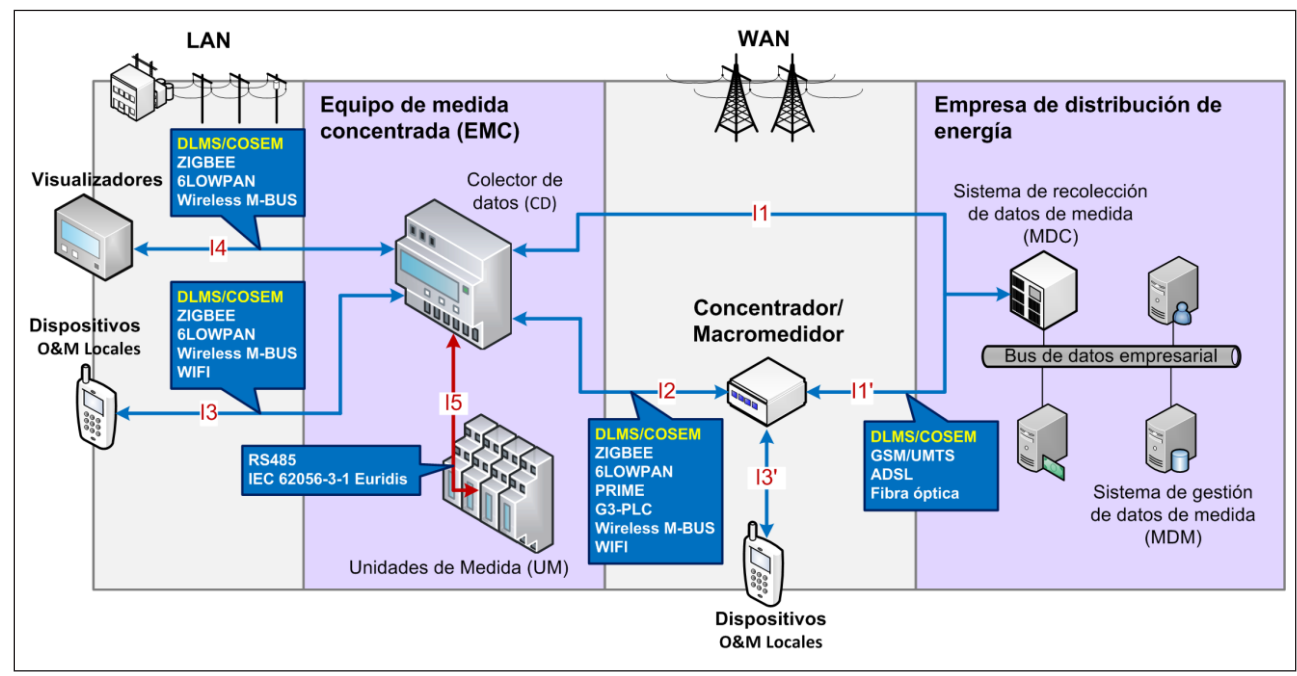

Figura 5. Propuesta de estándares.

Fuente: elaboración propia

no técnicas de energía no les genera un impacto notable que requiera la aplicación de este tipo de soluciones. Por tal razón, la selección de un estándar apropiado para los SMC se convierte en un proceso de investigación y análisis, que tiene en cuenta los aspectos de aplicación, seguridad, privacidad, robustez, eficiencia y costos. Los resultados de este artículo son, por lo tanto, una guía que les permite a las empresas de servicios públicos escoger de forma objetiva las tecnologías y estándares que mejor les convengan, según su arquitectura de comunicaciones, para la implementación de SMC que cumplan satisfactoriamente sus requerimientos.

El valor que tienen los estándares y tecnologías propuestas para SMC radica en que, además de cumplir los requerimientos planteados, facilitan la interoperabilidad por encima de otras soluciones propietarias como TWACS. Dicha interoperabilidad está libre de inversiones adicionales encaminadas al acondicionamiento de redes de comunicaciones, aplicaciones y bases de datos, así como de adquirir nuevo Software para la recolección de información en múltiples protocolos.

El uso de tecnologías con gran penetración en el mercado y aplicadas sobre varias interfaces puede ayudar a disminuir considerablemente los costos de implementación. WIFI; por ejemplo, al contar con mayor presencia que las tecnologías PRIME y G3PLC, se vuelve favorable para su utilización en I 3 e I2, siempre y cuando los equipos estén instalados en un poste y no en una base o subterráneo.

Los SMC son sistemas complejos que integran distintos elementos como medidores físicos, colectores de datos, concentradores, visualizadores y diversos módulos 
de comunicación. La elección de DLMS/COSEM, para este tipo de sistemas, presenta una mayor ventaja, comparando con otros estándares, debido a su forma natural de modelar dispositivos complejos utilizando un enfoque basado en objetos. Las estructuras de datos compuestas por tablas, aunque son eficientes y gozan de un nivel de sobrecarga de procesamiento mucho menor, son menos flexibles y requieren de una planeación cuidadosa para que su implementación en los SMC sea escalable y de fácil mantenimiento.

\section{CONCLUSIONES}

Se planteó una metodología para clasificar, seleccionar y proponer un conjunto de estándares, para su aplicación sobre SMC, basada en una serie de requerimientos con el nivel de tecnologías y protocolos y que puede ser extendida a cualquier sistema inteligente dentro de AMI.

Seleccionar DLMS/COSEM, sobre otros estándares, facilita que los componentes de la arquitectura AMI intercambien información sin depender del canal de comunicación; esto se debe a que DLMS/COSEM define en su implementación el soporte de las tecnologías más fundamentales y comunes en AMI, lo que posibilita, además, su aplicación sobre SMC, a pesar de que el estándar no haya sido orientado en principio a este tipo de soluciones.

\section{AGRADECIMIENTOS}

Los autores expresan agradecimientos al grupo Percepción y Sistemas Inteligentes, de la Universidad del Valle, por el apoyo en la realización de este trabajo.

\section{REFERENCIAS BIBLIOGRÁFICAS}

[1] E. Caicedo, B. Calvache, A. Barandica, O. Polanco, E. León, S. Medina, L. F. Tobón, and E. Tobón, "Una nueva solución para la gestión integral de energía eléctrica en sistemas de distribución,” presentado en Investigación y Desarrollo - Premio ASOCODIS - CNO, 2008.

[2] IEEE, IEEE Standard for Local Area Network/Wide Area Network (LAN/WAN) Node Communication Protocol to Complement the Utility Industry End Device Data Tables, IEEE Std 1703-2012, pp. 1-239, June 2012.

[3] DLMS UA, Architecture and Protocols, DLMS User Association Standard, 2009.

[4] G. Mauri, D. Moneta, G. Kmethy, G. Fantini, W. Strabbing, W. Freudenberg, H. Hohl, T. Schaub, T. Gerbaulet, H.-W. Kneitinger, T. Wiedemann, G. Radtke, F. Tarruell, and J. Comabella, "STATE-OF-THE-ART TECHNOLOGIES AND PROTOCOLS - Description of State of the Art Communication Protocols and Data Structures," The OPEN meter Consortium, Inf. téc., 2010. 
[5] IEEE, IEEE Standard for Utility Industry Metering Communication Protocol Application Layer (End Device Data Tables), IEEE Std 1377-2012 (Revision of IEEE Std 1377-1997), pp. 1-576, Aug 2012.

[6] IEEE, IEEE Standard for Optical Port Communication Protocol to Complement the Utility Industry End Device Data Tables, IEEE Std 1701-2011, pp. 1-50, Feb 2011.

[7] S. Liaisons, R. Hall, M. Modera, C. Neilson, B. Isler, M. Osborne, D. P. Alexander, C. L. Brumley, C. H. Copass, S. E. Dinges, S. G. Donaldson, D. G. Holmberg, D. Kollodge, T. Kurowski, B. Meyers, H. M. Newman, F. Schubert, G. M. Spiro, D. B. Thompson, K. Wagner, G. N. Wichenko, C. Zeller, E. C. Bryant, J. Butler, R. Bykowski, D. Fisher, N.-g. Fritz, C. Gemmill, D. P. Giorgis, J. Hartman, T. T. Heikkil, C. Jones, S. Karg, S. Lemaire, and J. Lenart, BACnet - A Data Communication Protocol for Building Automation and Control Networks, ANSI/ASHRAE Standard 135, 2012.

[8] S. H. Hong, S. H. Kim, G. M. Kim, and H. L. Kim, "Experimental evaluation of BZ-GW (BACnet-ZigBee smart grid gateway) for demand response in buildings," Energy, vol. 65, pp. 62-70, feb 2014. [Online]. Available: http://linkinghub.elsevier.com/retrieve/pii/S0360544213010657

[9] IEC, "Distribution automation using distribution line carrier systems - Part 5-1: Lower layer profiles - The spread frequency shift keying (S-FSK) profile," International Electrotechnical Commission, Standard IEC 61334-5-1, 2002. [En línea]. Disponible: https://webstore.iec.ch/ preview/info_iec61334-5-1\%7Bed2.0\%7Db.pdf

[10] IEC, "Communication networks and systems for power utility automation-ALLPARTS," International Electrotechnical Commission, Standard, 2015.

[11] IEC, "Electricity metering - Data exchange for meter reading, tariff and load control - Part 21: Direct local data exchange," International Electrotechnical Commission, Standard IEC 62056-21, 2002. [En línea]. Disponible: http://webstore.iec.ch/preview/info_iec62056-21\{ed1.0\} en_d.pdf

[12] IEC, "Electricity metering data exchange - The DLMS/COSEM suite - Part 3-1: Use of local area networks on twisted pair with carrier signalling," International Electrotechnical Commission, Standard IEC 62056-3-1:2013, 2013.

[13] IEC, "Electricity metering data exchange - The DLMS/COSEM suite - Part 1-0: Smart metering standardisation framework," International Electrotechnical Commission, Standard IEC 62056-1-0, 2014.

[14] CEN, “Communication systems for meters - Part 1: Data exchange," CEN-CENELEC, Standard EN 13757-1, 2015.

[15] Modbus, "Modbus Application Protocol Specification V1.1b3," Modbus Organization, Inf. téc., 2012.

[16] Landis, Wisy, and Dr. Neuhaus, “Smart Message Language Version 1.03," DKE, Standard $1.03,2008$. 
[17] CENELEC, "Electricity metering data exchange - Part 4: Lower layer PLC profile using SMITP B-PSK modulation,” CEN-CENELEC, Standard CLC/TS 50568-4:2015, 2015.

[18] P. PRIME, "PRIME v1.4 Specifications,” PRIME Alliance, Standard, 2014. [En línea]. Disponible: http://www.prime-alliance.org/?p=3470

[19] ZigBee, "Smart Energy Profile 2 Application Protocol Standard," ZigBee Alliance, Inf. téc., 2013.

[20] G. Montenegro, N. Kushalnagar, J. Hui, and D. Culler, “Transmission of IPv6 Packets over IEEE 802.15.4 Networks,” RFC Editor, RFC 4944, 2007, [En línea] Disponible: https://tools. ietf.org $/ \mathrm{html} / \mathrm{rfc} 4944$

[21] J. Hui and P. Thubert, "Compression Format for IPv6 Datagrams over IEEE 802.15.4-Based Networks,” RFC Editor, RFC 6282, 2011. [En línea]. Disponible: https://tools.ietf.org/html/ rfc6282

[22] Z. Shelby, S. Chakrabarti, E. Nordmark, and C. Bormann, "Neighbor Discovery Optimization for IPv6 over Low-Power Wireless Personal Area Networks (6LoWPANs)," RFC Editor, RFC 6775, 2012. [En línea]. Disponible: https://tools.ietf.org/html/rfc6775

[23] DLT, “DL/T 645 - 2007 Multi-Function Watt-Hour Meter Communications Protocol,” People’s Republic of China, Inf. téc., 2007.

[24] ERDF, "Electricity metering - Data exchange over powerline - Part 2 : Lower layer profile using OFDM modulation type 2,” CENELEC, Inf. téc., 2011.

[25] ITU-T, "Unified high-speed wireline-based home networking transceivers - System architecture and physical layer specification," International Telecommunication Union, Standard G.9960, 2015.

[26] M. Kuzlu, M. Pipattanasomporn, and S. Rahman, "Communication network requirements for major smart grid applications in HAN, NAN and WAN," Computer Networks, vol. 67, pp. 74-88, 2014. [En línea]. Disponible: http://www.sciencedirect.com/science/article/pii/ S1389128614001431

[27] B. Karimi, V. Namboodiri and M. Jadlwala, "Scalable Meter Data Collection in Smart Grids Through Message Concatenation”, IEEE Transactions on Smart Grid, vol 6, pp 1697-1706, 2015. [En línea]. Disponible: http://ieeexplore.ieee.org/stamp/ 